\title{
Fixed-Time Stability Analysis of Permanent Magnet Synchronous Motors with Novel Adaptive Control
}

\author{
Maoxing Liu, ${ }^{1}$ Jie Wu, ${ }^{2}$ and Yong-zheng Sun ${ }^{2}$ \\ ${ }^{1}$ Department of Mathematics, North University of China, Taiyuan 030051, China \\ ${ }^{2}$ School of Mathematics, China University of Mining and Technology, Xuzhou 221116, China \\ Correspondence should be addressed to Maoxing Liu; liumxsx@gmail.com
}

Received 6 September 2017; Revised 21 November 2017; Accepted 5 December 2017; Published 25 December 2017

Academic Editor: Alessandro Lo Schiavo

Copyright (C) 2017 Maoxing Liu et al. This is an open access article distributed under the Creative Commons Attribution License, which permits unrestricted use, distribution, and reproduction in any medium, provided the original work is properly cited.

\begin{abstract}
We firstly investigate the fixed-time stability analysis of uncertain permanent magnet synchronous motors with novel control. Compared with finite-time stability where the convergence rate relies on the initial permanent magnet synchronous motors state, the settling time of fixed-time stability can be adjusted to desired values regardless of initial conditions. Novel adaptive stability control strategy for the permanent magnet synchronous motors is proposed, with which we can stabilize permanent magnet synchronous motors within fixed time based on the Lyapunov stability theory. Finally, some simulation and comparison results are given to illustrate the validity of the theoretical results.
\end{abstract}

\section{Introduction}

In 1963, American meteorologist Edward Lorenz firstly explored chaotic phenomena [1]. After that, chaotic behavior has been extensively investigated in many fields such as medical science, biological engineering, secure communication, and engineering science [2-4]. Past few decades witnessed widely the chaos controlling and synchronization in various aspects such as information processing, salt-water oscillators, biological system, semiconductor lasers, power electronics, and chemical reactions [5-10]. But in 1989, chaos phenomenon was firstly investigated in the motor drive systems by Kuroe and Hayashi [11]. In the mid 1990s, Hemati discovered the chaos phenomena of the open-loop system of permanent magnet motor [12]. Later on, the mathematical model of the permanent magnet synchronous motor (PMSM) was first derived and the dynamic characteristics were studied in [13].

In fact, the emergence of chaos, as an undesirable phenomenon, may lead to the instability of control performance, the violent oscillation of the torque or speed, irregular electromagnetic noise, and even collapse of the PMSM. It is well known that PMSM plays a very important role in the process of industrial production. Therefore, it is necessary to control chaos behavior for eliminating the undesired performance
[14] which has attracted more and more consideration in the area of linear and nonlinear control [15]. The classical Lyapunov exponent methods were adopted for eliminating the chaos in PMSM [16, 17]. Harb investigated the nonlinear sliding mode control for eliminating the chaotic behavior in the PMSM [18]. Both of Choi and Maeng explored the adaptive control of a chaotic PMSM [19, 20]. Loria developed the robust linear control of chaotic PMSM with uncertainties [21] and further extended the adaptive linear control in PMSM [22]. And other control protocols were employed extensively including backstepping control [23], model predictive control [24], sensorless control [25], and others [26].

In those aforementioned strategies, asymptotic stabilities of chaotic PMSM systems are guaranteed only when time goes to the infinity. But from the practical engineering point of view and the perspective of the optimal time, it is full of significance to stabilize chaotic PMSM systems in a finite time. Recently, many researchers developed the finite-time stable control and synchronization of the chaotic PMSM system. In [27], the authors studied the controlling chaos in PMSM based on finite-time stability theory. In [28], the authors discussed the finite-time stability control of chaotic PMSM system with parameters uncertain. In [29], the authors further considered the finite-time stabilization problem to eliminate the chaos in PMSM by adopting adaptive control. 
A robust finite-time chaos synchronization scheme was proposed for two uncertain third-order PMSMs [30]. Sun et al. investigated the finite-time synchronization control and parameter identification of uncertain PMSM with a novel adaptive control scheme [31]. Besides these, the finite-time control scheme has been extensively harnessed in other areas, such as high-order nonholonomic mobile robots [32], and multiagent systems [33].

We can date back to the 1960 for finding the concept of the finite-time stability. We know that the key point in finite-time results is that the power exponent of the Lyapunov function is less than one. The convergence rate of the finite-time results depends on the initial conditions $i_{d}(0), i_{q}(0), \omega(0)$. Different initial direct-axis current $i_{d}$, quadrature-axis current $i_{q}$, and angular frequency $\omega$ may result in different convergence time. However, the initial conditions of some practical systems can hardly be adjusted or estimated, which leads to the inaccessibility of the final settling time and deteriorating of the system's performance. To overcome this drawback, Polyakov [34] introduced a nonlinear feedback design for the fixed-time stabilization of linear systems, where the definition of fixed-time stable was firstly proposed. Later on, further investigations of fixed-time consensus and stabilization problems have also been presented $[35,36]$.

Inspired by the above analysis, this paper firstly explores the fixed-time stability analysis of uncertain PMSM by employing adaptive control, which can accelerate the convergence rate independent of the initial conditions. This has not been investigated in the existing literature, which is actually the main contribution of this paper. Different from the previous study [27-31] concerning the finite-time stability or synchronization, where the final convergence time is closely related to the initial conditions, the settling time of the fixed-time stability can be directly calculated and predesigned regardless of the initial state of the PMSM. And we can obtain a faster convergent speed than usual, which will be verified in simulation.

The remainder of this paper is as follows. In Section 2, we introduce the model description and problem formulation. Section 3 gives the basic conception of fixed-time stability. Section 4 discusses the adaptive fixed-time stability of uncertain permanent magnet synchronous motors with novel control. In Section 5, numerical simulations are performed to verify the feasibility and effectiveness of the analytical results. Finally, we will give some conclusions in Section 6.

\section{Model Description and Problem Formulation}

In general, the dimensionless mathematical model of PMSM with the smooth air gap is considered [13]:

$$
\begin{aligned}
& \frac{\mathrm{d} i_{d}}{\mathrm{~d} t}=-i_{d}+i_{q} \omega+\widetilde{u}_{d}, \\
& \frac{\mathrm{d} i_{q}}{\mathrm{~d} t}=-i_{q}-i_{d} \omega+\gamma \omega+\widetilde{u}_{q}, \\
& \frac{\mathrm{d} \omega}{\mathrm{d} t}=\sigma\left(i_{q}-\omega\right)-\widetilde{T}_{L},
\end{aligned}
$$

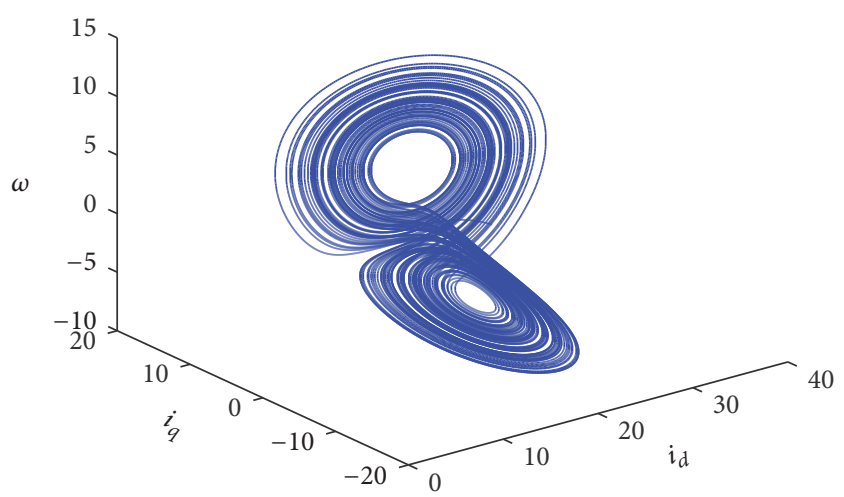

FIGURE 1: Chaotic attractor generated by the system $(2)$ when $i_{d}(0)=$ $25, i_{q}(0)=1, \omega(0)=-1, \sigma=5.46, \gamma=20$ in 3-dimension space.

where $i_{d}, i_{q}$, and $\omega$ are the state variables which denote the $d$-axis and $q$-axis stator currents and angle speed of the motor, respectively; $\tilde{u}_{d}$ and $\tilde{u}_{q}$ are the $d$-axis and $q$-axis stator voltages, respectively; $\widetilde{T}_{L}$ is external load torque; $\sigma>0$ and $\gamma>0$ are the system operating parameters.

The external inputs $\widetilde{u}_{d}, \widetilde{u}_{q}$, and $\widetilde{T}_{L}$ are set to be zero after an operating period of the system. Then the unforced system (1) becomes

$$
\begin{aligned}
& \frac{\mathrm{d} i_{d}}{\mathrm{~d} t}=-i_{d}+i_{q} \omega, \\
& \frac{\mathrm{d} i_{q}}{\mathrm{~d} t}=-i_{q}-i_{d} \omega+\gamma \omega, \\
& \frac{\mathrm{d} \omega}{\mathrm{d} t}=\sigma\left(i_{q}-\omega\right) .
\end{aligned}
$$

The bifurcation and chaos phenomenon of the above system (2) are investigated fully in [2-7]. It has been found that the permanent magnet synchronous motor is experiencing chaotic behavior with the initial conditions of system (2) and the operating parameters $\sigma$ and $\gamma$ falling into a certain area. For example, the typical chaotic behavior can be observed in Figure 1 given that $\tilde{u}_{d}(0)=0, \widetilde{u}_{q}(0)=0, \widetilde{T}_{L}(0)=0, \sigma=$ $5.46, \gamma=20$ and the initial conditions are $i_{d}(0)=25, i_{q}(0)=$ $1, \omega(0)=-1$, which appear as aperiodic, reciprocating, sudden, or intermittent morbid oscillations of the motor angle speed.

As we all know, chaotic behavior in PMSM can seriously destroy the stable operation of the motor, even leading to industrial driven system collapse. Therefore, it is very necessary to steer the direct-axis current $i_{d}$, quadrature-axis current $i_{q}$, and angle speed $\omega$ to arrive at the stable state for eliminating the chaos within fixed time by designing appropriate controllers in PMSM, which is full of significance and is the main purpose of our investigation.

\section{Basic Conception of Fixed-Time Stability}

In this paper, we investigate the fixed-time chaos controlling in PMSM by employing an adaptive controller. For ease of 
analysis, we give some necessary definition and lemmas in advance.

Definition 1. Consider the following nonlinear dynamic system:

$$
\dot{x}=f(x),
$$

where $x \in R^{n}$ is the system state and $f$ is a smooth nonlinear function. If there exists a settling time $T$ independent of the initial condition $x(0)=x_{0}$, such that

$$
\lim _{t \rightarrow T}\|x(t)\|=0
$$

and $\|x(t)\| \equiv 0$ for all $t \geq T$, then system (3) is fixed-time stable.

Lemma 2 (see [37]). Supposing there exists a continuous function $v(t):=V(x(t)):[0, \infty) \rightarrow[0, \infty)$, such that

(1) $v(t)$ is positive definite;

(2) there exist real numbers $c>0$ and $0<\rho<1$ such that

$$
\dot{v}(t) \leq-c v^{\rho}(t),
$$

then one has $v(t) \equiv 0, t \geq T$, where the setting time, depending on the initial state $x_{0}$, satisfies

$$
T \leq \frac{v^{1-\rho}\left(x_{0}\right)}{c(1-\rho)} .
$$

Lemma 3 (see [34]). If there exists a continuous radically unbounded function $V: R^{n} \rightarrow R_{+} \cup|0|$ such that

(1) $V(x(t))=0 \Leftrightarrow x(t)=0$;

(2) any solution $x(t)$ satisfies the inequality $D^{*} V(x(t)) \leq$ $-\left(\alpha V^{p}(x(t))+\beta V^{q}(x(t))\right)^{k}$ for $\alpha, \beta, p, q, k>0$, pk $<1$, and $q k>1$, where $D^{*} V(x(t))$ denotes the upper right hand derivative of the function $V(x(t))$, then the origin is globally fixed-time stable and the following estimate holds:

$$
T \leq \frac{1}{\alpha^{k}(1-p k)}+\frac{1}{\beta^{k}(q k-1)} .
$$

Lemma 3 presents quite a conservative settling time estimate. A more accurate estimate is provided in the next lemma. Consider the case where the constants $p$ and $q$ are of the form $p=1-1 / 2 \gamma$ and $q=1+1 / 2 \gamma, \gamma>1$.

Lemma 4 (see [34]). If there exists a continuous radically unbounded function $V: R^{n} \rightarrow R_{+} \cup|0|$ such that

(1) $V(x(t))=0 \Leftrightarrow x(t)=0$;

(2) any solution $x(t)$ satisfies the inequality $D^{*} V(x(t)) \leq$ $-\alpha V^{p}(x(t))-\beta V^{q}(x(t))$ for some $\alpha, \beta, p=1-1 / 2 \gamma, q=$ $1+1 / 2 \gamma$, and $\gamma>1$, where $D^{*} V(x(t))$ denotes the upper right hand derivative of the function $V(x(t))$, then the origin is globally fixed time stable and the following estimate holds:

$$
T \leq T_{\max }:=\frac{\pi \gamma}{\sqrt{\alpha \beta}} .
$$

Lemma 5 (see [38]). If $x_{1}, x_{2}, \ldots, x_{N} \geq 0$, then

$$
\sum_{i=1}^{N} x_{i}^{\eta} \geq\left(\sum_{i=1}^{N} x_{i}\right)^{\eta}, \quad 0<\eta \leq 1,
$$

$$
\sum_{i=1}^{N} x_{i}^{\varsigma} \geq N^{1-\varsigma}\left(\sum_{i=1}^{N} x_{i}\right)^{\varsigma}, \quad \varsigma>1 .
$$

\section{Main Results}

For achieving fixed-time stability in PMSM, we implement the controls $u_{1}, u_{2}$, and $u_{3}$ to system (2), then the controlled system can be described by

$$
\begin{aligned}
& \frac{\mathrm{d} i_{d}}{\mathrm{~d} t}=-i_{d}+i_{q} \omega+u_{1}, \\
& \frac{\mathrm{d} i_{q}}{\mathrm{~d} t}=-i_{q}-i_{d} \omega+\gamma \omega+u_{2}, \\
& \frac{\mathrm{d} \omega}{\mathrm{d} t}=\sigma\left(i_{q}-\omega\right)+u_{3} .
\end{aligned}
$$

Now, we will design appropriate controllers $u_{1}, u_{2}, u_{3}$ for realizing the global stability of the above PMSM system based on the fixed-time stability theory, where the system can settle to the equilibrium $x^{*}=\left(i_{d}^{*}, i_{q}^{*}, \omega^{*}\right)=(0,0,0)$ regardless of the initial state $i_{d}(0), i_{q}(0), \omega(0)$. For other equilibria, one can also apply this method presented below to discuss the stability analysis. According to the above analysis, we give our main results in the following theorem.

Theorem 6. Let $g_{1}>0, g_{2}>0$, and $g_{3}>0$ be arbitrarily chosen constants. System (10) in closed-loop with the controllers

$$
\begin{aligned}
& u_{1}=-k_{1} i_{d}^{\alpha}-k_{1} i_{d}^{\beta}, \\
& u_{2}=-k_{2} i_{q}^{\alpha}-k_{2} i_{q}^{\beta}, \\
& u_{3}=-\sigma i_{q}-k_{3} \omega^{\alpha}-k_{3} \omega^{\beta},
\end{aligned}
$$

where

$$
\begin{aligned}
& \dot{k}_{1}=i_{d}^{\alpha+1}+i_{d}^{\beta+1}-\left(k_{1}-g_{1}\right)^{\alpha}-\left(k_{1}-g_{1}\right)^{\beta}, \\
& \dot{k}_{2}=i_{q}^{\alpha+1}+i_{q}^{\beta+1}-\left(k_{2}-g_{2}\right)^{\alpha}-\left(k_{2}-g_{2}\right)^{\beta}, \\
& \dot{k}_{3}=\omega^{\alpha+1}+\omega^{\beta+1}-\left(k_{3}-g_{3}\right)^{\alpha}-\left(k_{3}-g_{3}\right)^{\beta},
\end{aligned}
$$

is globally fixed-time stable for any $0<\alpha<1, \beta>1$.

Proof. We divide the process of proof into two steps.

In the first step, we select the Lyapunov candidate function

$$
V_{1}(x)=\frac{1}{2} \omega^{2}+\frac{1}{2}\left(k_{3}-g_{3}\right)^{2} .
$$

The derivative along the trajectory of the third subsystem in (10) gives

$$
\begin{aligned}
\dot{V}_{1} & =\omega \dot{\omega}+\left(k_{3}-g_{3}\right) \dot{k}_{3} \\
& =\omega\left[\sigma\left(i_{q}-\omega\right)+u_{3}\right]+\left(k_{3}-g_{3}\right) \dot{k}_{3} .
\end{aligned}
$$


By adopting the designed controller $u_{3}$ of the system in (11) and the corresponding updating law of the third subsystem in (12), we get

$$
\begin{aligned}
\dot{V}_{1} & =\omega\left[\sigma\left(i_{q}-\omega\right)-\sigma i_{q}-k_{3} \omega^{\alpha}-k_{3} \omega^{\beta}\right]+\left(k_{3}-g_{3}\right) \\
\cdot & {\left[\omega^{\alpha+1}+\omega^{\beta+1}-\left(k_{3}-g_{3}\right)^{\alpha}-\left(k_{3}-g_{3}\right)^{\beta}\right]=-\sigma \omega^{2} } \\
& -g_{3} \omega^{\alpha+1}-g_{3} \omega^{\beta+1}-\left(k_{3}-g_{3}\right)^{\alpha+1}-\left(k_{3}-g_{3}\right)^{\beta+1} \\
& \leq-g_{3} \omega^{\alpha+1}-g_{3} \omega^{\beta+1}-\left(k_{3}-g_{3}\right)^{\alpha+1}-\left(k_{3}\right. \\
& \left.-g_{3}\right)^{\beta+1}=-2^{(1 / 2)(\alpha+1)} g_{3}\left(\frac{1}{2} \omega^{2}\right)^{(1 / 2)(\alpha+1)} \\
& -2^{(1 / 2)(\alpha+1)}\left(\frac{1}{2}\left(k_{3}-g_{3}\right)^{2}\right)^{(1 / 2)(\alpha+1)} \\
& -2^{(1 / 2)(\beta+1)} g_{3}\left(\frac{1}{2} \omega^{2}\right)^{(1 / 2)(\beta+1)} \\
& -2^{(1 / 2)(\beta+1)}\left(\frac{1}{2}\left(k_{3}-g_{3}\right)^{2}\right)^{(1 / 2)(\beta+1)} \\
& \leq-m_{1}\left[\left(\frac{1}{2} \omega^{2}\right)^{(1 / 2)(\alpha+1)}\right. \\
& \left.+\left(\frac{1}{2}\left(k_{3}-g_{3}\right)^{2}\right)^{(1 / 2)(\alpha+1)}\right]-n_{1}\left[\left(\frac{1}{2} \omega^{2}\right)^{(1 / 2)(\beta+1)}\right. \\
& \left.+\left(\frac{1}{2}\left(k_{3}-g_{3}\right)^{2}\right)^{(1 / 2)(\beta+1)}\right]
\end{aligned}
$$

where $m_{1}=\min \left\{2^{(1 / 2)(\alpha+1)} g_{3}, 2^{(1 / 2)(\alpha+1)}\right\}, n_{1}=\min \left\{2^{(1 / 2)(\beta+1)} g_{3}\right.$, $\left.2^{(1 / 2)(\beta+1)}\right\}$.

Thus, it follows from Lemma 5 that

$\dot{V}_{1}$

$$
\begin{aligned}
\leq & -m_{1}\left[\left(\frac{1}{2} \omega^{2}\right)+\left(\frac{1}{2}\left(k_{3}-g_{3}\right)^{2}\right)\right]^{(1 / 2)(\alpha+1)} \\
& -2^{(1-\beta) / 2} n_{1}\left[\left(\frac{1}{2} \omega^{2}\right)+\left(\frac{1}{2}\left(k_{3}-g_{3}\right)^{2}\right)\right]^{(1 / 2)(\beta+1)} \\
= & -m_{1} V_{1}^{(1 / 2)(\alpha+1)}-2^{(1-\beta) / 2} n_{1} V_{1}^{(1 / 2)(\beta+1)} .
\end{aligned}
$$

Thus, it follows from Lemma 3 that the third subsystem in (10) is stable in fixed time

$$
T_{1} \leq \frac{2}{m_{1}(1-\alpha)}+\frac{2^{(\beta+1) / 2}}{n_{1}(\beta-1)},
$$

which means that $\omega \equiv 0$ and $k_{3} \equiv g_{3}$ when $t \geq T_{1}$.

In the second step, when $t \geq T_{1}, \omega=0$, we can obtain the following subsystem:

$$
\begin{aligned}
& \frac{\mathrm{d} i_{d}}{\mathrm{~d} t}=-i_{d}+u_{1}, \\
& \frac{\mathrm{d} i_{q}}{\mathrm{~d} t}=-i_{q}+u_{2} .
\end{aligned}
$$

Then, we select the following Lyapunov candidate function:

$$
V_{2}(x)=\frac{1}{2} i_{d}^{2}+\frac{1}{2} i_{q}^{2}+\frac{1}{2}\left(k_{1}-g_{1}\right)^{2}+\frac{1}{2}\left(k_{2}-g_{2}\right)^{2} .
$$

The derivative along the trajectory of the subsystem in (18) by adopting the adaptive controllers $u_{1}$ and $u_{2}$ gives

$$
\begin{aligned}
& \dot{V}_{2}=i_{d}\left[-i_{d}+u_{1}\right]+i_{q}\left[-i_{q}+u_{2}\right]+\left(k_{1}-g_{1}\right)\left[i_{d}^{\alpha+1}\right. \\
& \left.+i_{d}^{\beta+1}-\left(k_{1}-g_{1}\right)^{\alpha}-\left(k_{1}-g_{1}\right)^{\beta}\right]+\left(k_{2}-g_{2}\right)\left[i_{q}^{\alpha+1}\right. \\
& \left.+i_{q}^{\beta+1}-\left(k_{2}-g_{2}\right)^{\alpha}-\left(k_{1}-g_{1}\right)^{\beta}\right]=-i_{d}^{2}-g_{1} i_{d}^{\alpha+1} \\
& -g_{1} i_{d}^{\beta+1}-i_{q}^{2}-g_{2} i_{q}^{\alpha+1}-g_{2} i_{q}^{\beta+1}-\left(k_{1}-g_{1}\right)^{\alpha+1} \\
& -\left(k_{1}-g_{1}\right)^{\beta+1}-\left(k_{2}-g_{2}\right)^{\alpha+1}-\left(k_{2}-g_{2}\right)^{\beta+1} \\
& \leq-2^{(1 / 2)(\alpha+1)} g_{1}\left(\frac{1}{2} i_{d}^{2}\right)^{(1 / 2)(\alpha+1)} \\
& -2^{(1 / 2)(\alpha+1)}\left(\frac{1}{2}\left(k_{1}-g_{1}\right)^{2}\right)^{(1 / 2)(\alpha+1)} \\
& -2^{(1 / 2)(\beta+1)} g_{1}\left(\frac{1}{2} i_{d}^{2}\right)^{(1 / 2)(\beta+1)} \\
& -2^{(1 / 2)(\beta+1)}\left(\frac{1}{2}\left(k_{1}-g_{1}\right)^{2}\right)^{(1 / 2)(\beta+1)} \\
& -2^{(1 / 2)(\alpha+1)} g_{2}\left(\frac{1}{2} i_{q}^{2}\right)^{(1 / 2)(\alpha+1)} \\
& -2^{(1 / 2)(\alpha+1)}\left(\frac{1}{2}\left(k_{2}-g_{2}\right)^{2}\right)^{(1 / 2)(\alpha+1)} \\
& -2^{(1 / 2)(\beta+1)} g_{2}\left(\frac{1}{2} i_{q}^{2}\right)^{(1 / 2)(\beta+1)} \\
& -2^{(1 / 2)(\beta+1)}\left(\frac{1}{2}\left(k_{2}-g_{2}\right)^{2}\right)^{(1 / 2)(\beta+1)} \\
& \leq-m_{2}\left[\left(\frac{1}{2} i_{d}^{2}\right)^{(1 / 2)(\alpha+1)}\right. \\
& \left.+\left(\frac{1}{2}\left(k_{1}-g_{1}\right)^{2}\right)^{(1 / 2)(\alpha+1)}\right]-n_{2}\left[\left(\frac{1}{2} i_{d}^{2}\right)^{(1 / 2)(\beta+1)}\right. \\
& \left.+\left(\frac{1}{2}\left(k_{1}-g_{1}\right)^{2}\right)^{(1 / 2)(\beta+1)}\right]-m_{2}\left[\left(\frac{1}{2} i_{q}^{2}\right)^{(1 / 2)(\alpha+1)}\right. \\
& \left.+\left(\frac{1}{2}\left(k_{2}-g_{2}\right)^{2}\right)^{(1 / 2)(\alpha+1)}\right]-n_{2}\left[\left(\frac{1}{2} i_{q}^{2}\right)^{(1 / 2)(\beta+1)}\right. \\
& \left.+\left(\frac{1}{2}\left(k_{2}-g_{2}\right)^{2}\right)^{(1 / 2)(\beta+1)}\right],
\end{aligned}
$$

where $m_{2}=\min \left\{2^{(1 / 2)(\alpha+1)} g_{1}, 2^{(1 / 2)(\alpha+1)} g_{2}, 2^{(1 / 2)(\alpha+1)}\right\}, n_{2}=$ $\min \left\{2^{(1 / 2)(\beta+1)} g_{1}, 2^{(1 / 2)(\beta+1)} g_{2}, 2^{(1 / 2)(\beta+1)}\right\}$. 
Thus, it follows from Lemma 5 that

$$
\begin{aligned}
\dot{V}_{2} \leq & -m_{2}\left[\left(\frac{1}{2} i_{d}^{2}\right)+\left(\frac{1}{2} i_{q}^{2}\right)+\left(\frac{1}{2}\left(k_{1}-g_{1}\right)^{2}\right)\right. \\
& \left.+\left(\frac{1}{2}\left(k_{2}-g_{2}\right)^{2}\right)\right]^{(1 / 2)(\alpha+1)}-2^{(1-\beta) / 2} n_{2}\left[\left(\frac{1}{2} i_{d}^{2}\right)\right. \\
& +\left(\frac{1}{2} i_{q}^{2}\right)+\left(\frac{1}{2}\left(k_{1}-g_{1}\right)^{2}\right) \\
& \left.+\left(\frac{1}{2}\left(k_{2}-g_{2}\right)^{2}\right)\right]^{(1 / 2)(\beta+1)} \\
= & -m_{2} V_{2}^{(1 / 2)(\alpha+1)}-2^{(1-\beta) / 2} n_{2} V_{2}^{(1 / 2)(\beta+1)} .
\end{aligned}
$$

Thus, it follows from Lemma 3 that $i_{d}$ and $i_{q}$ are stable in fixed time

$$
T_{2} \leq \frac{2}{m_{2}(1-\alpha)}+\frac{2^{(\beta+1) / 2}}{n_{2}(\beta-1)}
$$

which means that $i_{d} \equiv 0, i_{q} \equiv 0, k_{1} \equiv g_{1}$, and $k_{2} \equiv g_{2}$ when $t \geq T_{1}+T_{2}$.

Thus, by employing the proposed adaptive controller (11), we can make the PMSM stable within fixed time $T_{1}+T_{2}$. This completes the proof.

In order to obtain a more accurate estimate of convergence time, by adopting Lemma 4, we can get the following corollary.

Corollary 7. In protocol (11), if the parameters $\alpha$ and $\beta$ are selected as $\alpha=1-1 / \nu$ and $\beta=1+1 / \nu, \nu>1$, under the same conditions as Theorem 6 , the settling time of the third subsystem in (10) can be estimated as

$$
\widetilde{T}_{1} \leq T_{\text {max }_{1}}:=\frac{2^{(\beta-1) / 4} \pi \nu}{\sqrt{m_{1} n_{1}}}
$$

which means that $\omega \equiv 0$ and $k_{3} \equiv g_{3}$ when $t \geq \widetilde{T}_{1}$, and $i_{d}$ and $i_{q}$ are stable in fixed time

$$
\widetilde{T}_{2} \leq T_{\max _{2}}:=\frac{2^{(\beta-1) / 4} \pi \nu}{\sqrt{m_{2} n_{2}}}
$$

which means that $i_{d} \equiv 0, i_{q} \equiv 0, k_{1} \equiv g_{1}$, and $k_{2} \equiv g_{2}$ when $t \geq \widetilde{T}_{1}+\widetilde{T}_{2}$.

Thus, by employing the proposed adaptive controller (11), we can make the PMSM stable in fixed time within $\widetilde{T}_{1}+\widetilde{T}_{2}$.

Corollary 8. If we adopt the following protocol,

$$
\begin{aligned}
& u_{1}=-k_{1} i_{d}^{\alpha}, \\
& u_{2}=-k_{2} i_{q}^{\alpha}, \\
& u_{3}=-\sigma i_{q}-k_{3} \omega^{\alpha},
\end{aligned}
$$

where parameters $0<\alpha<1$, the feedback gains $k_{1}>0, k_{2}>0$, and $k_{3}>0$ which are the tuning parameters of the terminal attractor can be adapted according to the following update laws:

$$
\begin{aligned}
& \dot{k}_{1}=i_{d}^{\alpha+1}-\left(k_{1}-g_{1}\right)^{\alpha}, \\
& \dot{k}_{2}=i_{q}^{\alpha+1}-\left(k_{2}-g_{2}\right)^{\alpha}, \\
& \dot{k}_{3}=\omega^{\alpha+1}-\left(k_{3}-g_{3}\right)^{\alpha},
\end{aligned}
$$

where $g_{1}>0, g_{2}>0$, and $g_{3}>0$ are the arbitrary constants, respectively. By Lemma 2, we can estimate the finite time of the third subsystem in (10), which depends on the initial conditions of the PMSM as

$$
\widehat{T}_{1}=\frac{2 V_{1}^{(1-\alpha) / 2}(0)}{m_{1}(1-\alpha)},
$$

and $i_{d}$ and $i_{q}$ are stable in finite time

$$
\widehat{T}_{2}=\widehat{T}_{1}+\frac{2 V_{2}^{(1-\alpha) / 2}(0)}{m_{2}(1-\alpha)} .
$$

Remark 9. In summary, the past result of Theorem 1 in [31] is reflected as a special case, namely, Corollary 8, in our research. We have extended the finite-time stability to the fixed-time stability. Therefore, the obtained results in this paper are more general. In the future work, we will extend Theorem 2 [31], that is, finite-time synchronization and parameters identification, into fixed-time synchronization and parameters identification.

\section{Simulations Results}

In this section, an illustrative example is performed to verify the feasibility and effectiveness of the above analytical results. The method of numerical solutions, time step, the parameters, initial conditions, and the tuning parameters are the same as those in [31], where $\sigma=5.46, \gamma=20, \alpha=$ $7 / 9, i_{d}(0)=5, i_{q}(0)=1, \omega(0)=-1, k_{1}(0)=k_{2}(0)=k_{3}(0)=$ $0.2, g_{1}=1, g_{2}=1.5, g_{3}=2$.

Figure 2 shows the chaotic trajectories of the state variables $i_{d}, i_{q}$, $\omega$ without any control. Figure 3 shows the time response of the adaptive fixed-time controllers (11) which will settle to zero when the state variable $x=\left(i_{d}, i_{q}, \omega\right)$ converges to the equilibrium. Figure 4(a) shows the time response of the controlled PMSM state variables $i_{d}, i_{q}$, and $\omega$, which will arrive at the equilibrium within finite time. Figure 4(b) shows the tuning parameters of terminal attractors $k_{1}, k_{2}$, and $k_{3}$ which will converge to $g_{1}, g_{2}, g_{3}$, respectively.

For making a fair comparison with the recent result that is Theorem 1 in [31], the parameters, initial conditions, and the tuning parameters are the same as those in [31]. Figure 5 shows the convergent speed of state variable $i_{d}$ with fixedtime control in this paper and the speed of finite-time control in [31], respectively. Figure 6 shows the convergent speed of state variable $i_{q}$ with fixed-time control in this paper and the speed of finite-time control in [31], respectively. Figure 7 shows the convergent speed of state variable $\omega$ with fixed-time 

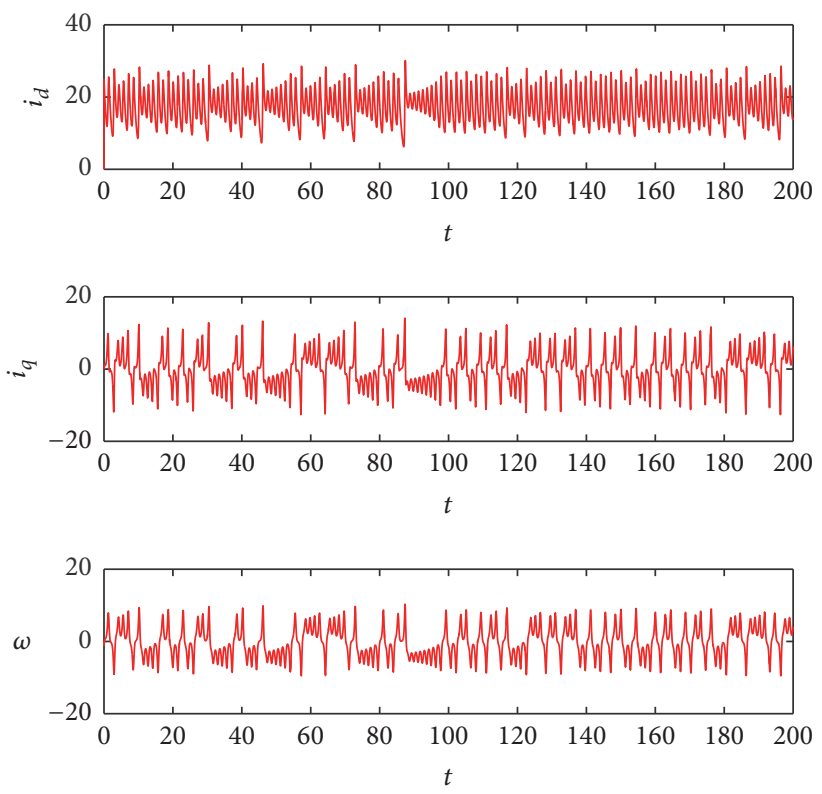

FIGURE 2: Chaotic trajectories of the state variables $i_{d}, i_{q}, \omega$ without any control.
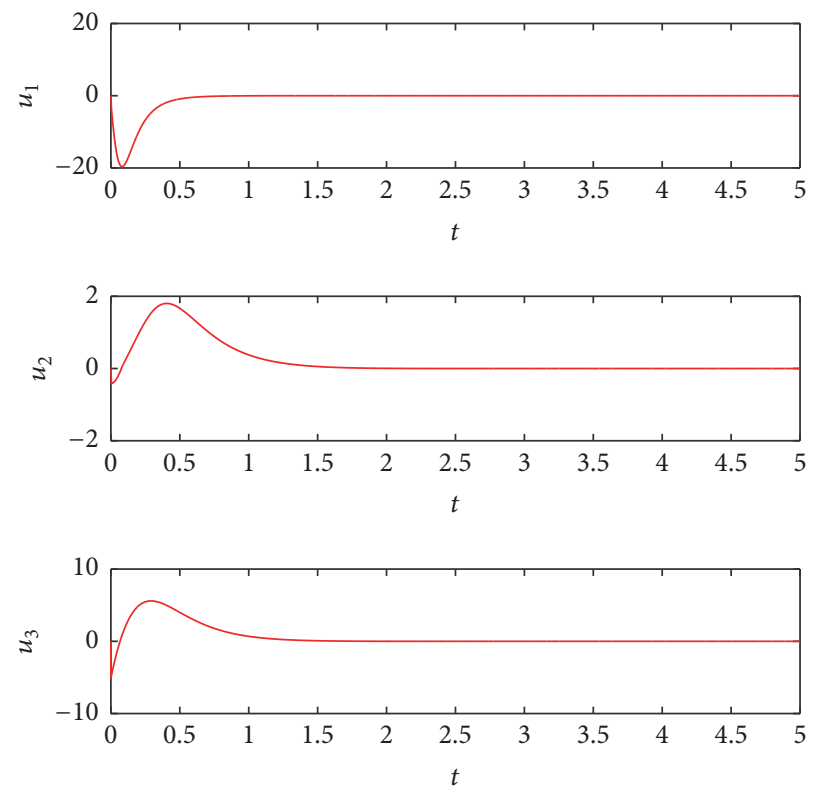

FIGURE 3: Time response of the adaptive fixed-time controllers $u_{1}, u_{2}$, and $u_{3}$.

control in this paper and the speed of finite-time control in [31], respectively. From Figures 5, 6, and 7, one can obtain that the fixed time needed for controlling chaos in our paper is shorter than finite time needed in [31]. Obviously, the time for controlling in this paper is too shorter than those in [2729] which had been compared in [31].

To explore the relationship between the convergent time and the value of the parameters $\alpha$ and $\beta$ experimentally, we select a representative variable $i_{q}$ to demonstrate the convergent time. Figure 8 gives the time response of $i_{q}$ with different values of $\alpha$. It is shown that the convergent time decreases when parameter $\alpha$ increases. Figure 9 gives the time response of $i_{q}$ with different values of $\beta$. It is shown that small $\beta$ can lead to a shorter convergence time than those with large $\beta$.

In order to verify that our proposed fixed-time stable theory is independent of the initial state, we take $\sigma=$ $5.46, \gamma=20, \alpha=7 / 9, \beta=1.1, g_{1}=1, g_{2}=1.5$, $g_{3}=2$, and the initial state $i_{d}(0), i_{q}(0), \omega(0)$ randomly from the larger interval $[-50,50]$ (other larger intervals are also permissible) for four times as shown in Figure 10. It is easy to find that all convergence times are no more than the upper bounds of theoretical settling time 29.7205 which is estimated by computing the sum of (17) and (22). Because 


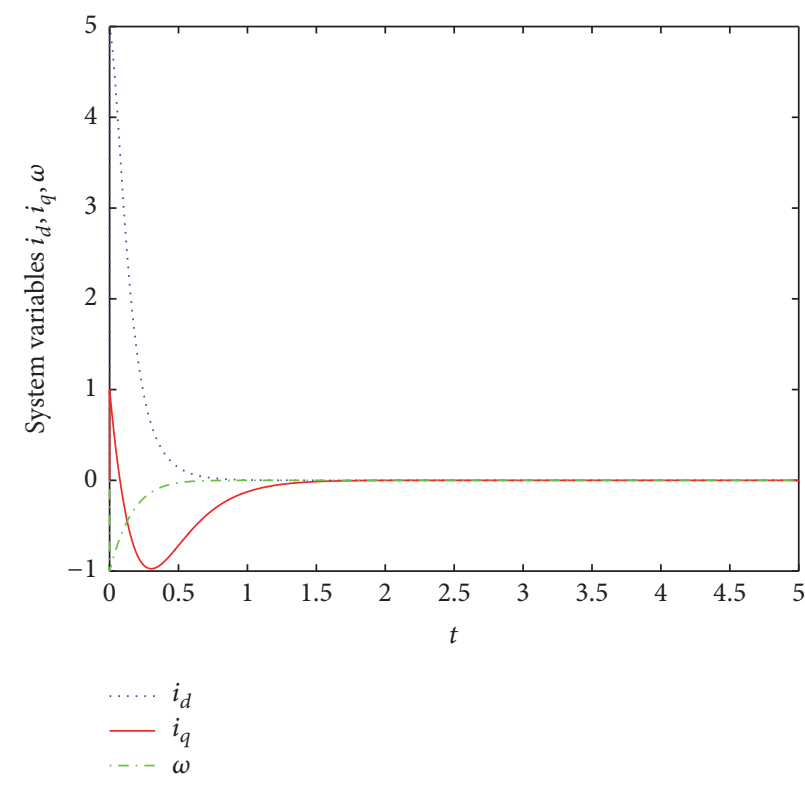

(a)

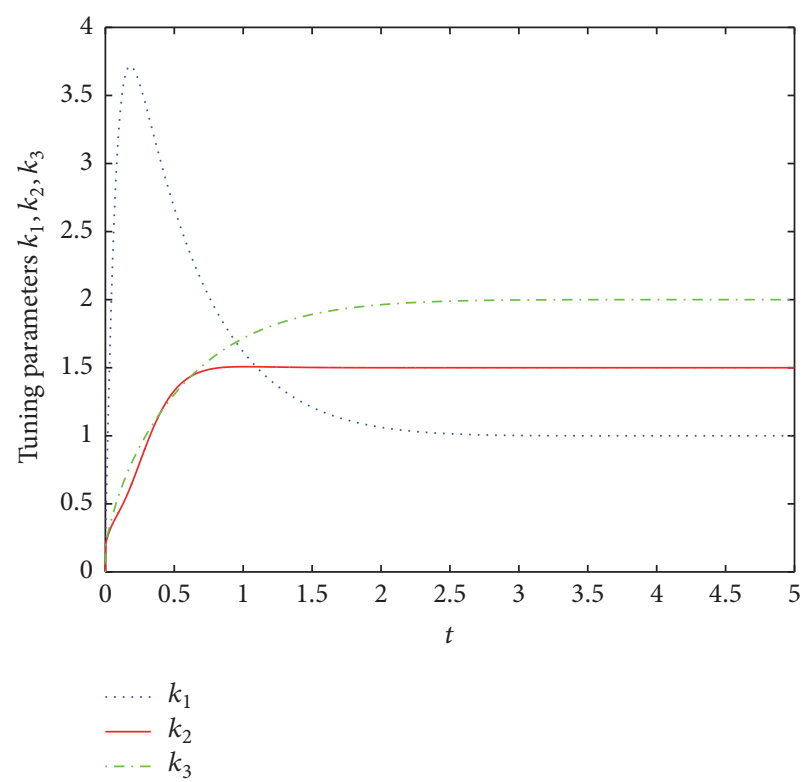

(b)

FIGURE 4: (a) Time response of the controlled state variables $i_{d}, i_{q}$, and $\omega$. (b) Tuning parameters of terminal attractors $k_{1}, k_{2}$, and $k_{3}$.

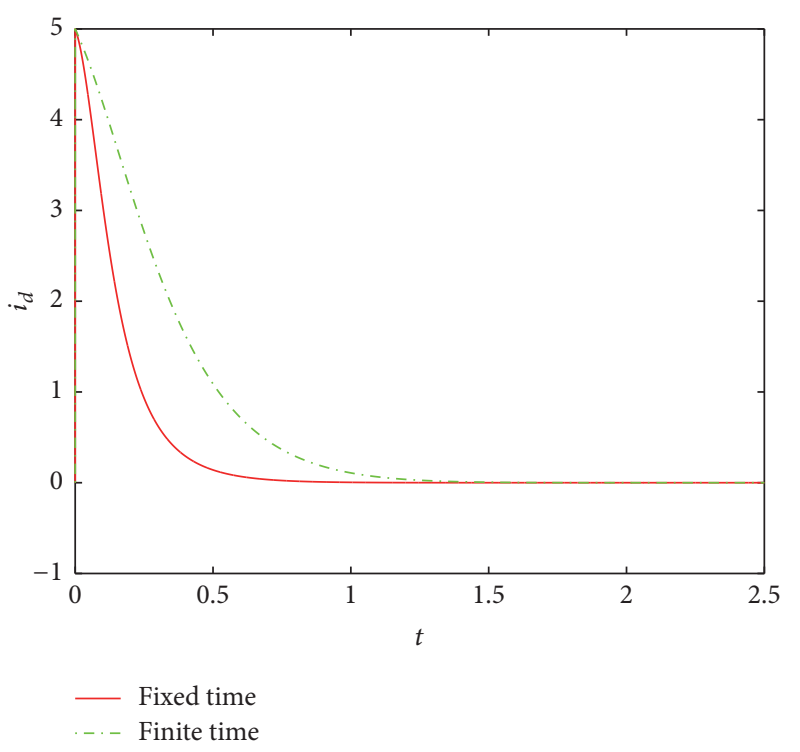

FIGURE 5: Comparison of convergent speed of $i_{d}$ in fixed time and finite time, respectively.

many inequalities are used in the proof of Theorem 6 and Lemmas 3 and 5, all practical convergence times around 2 observed from Figure 10 are less than the theoretical settling time.

In addition, we further test the disturbance rejection ability of the PMSM (10) with the designed control (11). We assume that the system state $x=\left(i_{d}, i_{q}, \omega\right)$ will suffer from the unknown injected disturbance $\left(\Delta i_{d}, \Delta i_{q}, \Delta \omega\right)$ at time $t=2$ (other time is also permissible) where each component is simulated by being randomly taken from the interval $[-10,10]$ (other larger intervals are also permissible)

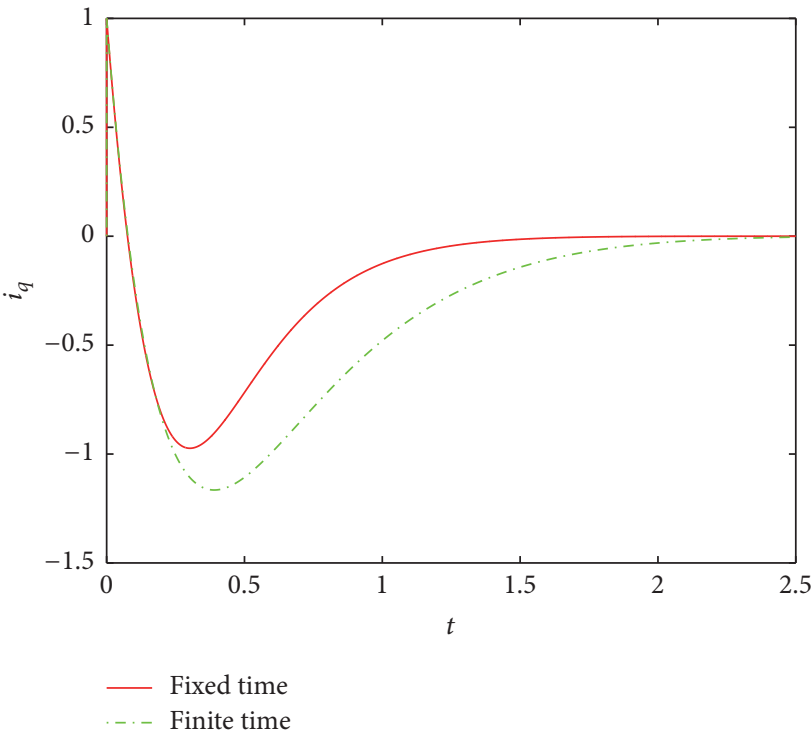

FIGURE 6: Comparison of convergent speed of $i_{q}$ in fixed time and finite time, respectively.

for two times. At this point, the disturbed state $x$ is $\left(i_{d}+\right.$ $\left.\Delta i_{d}, i_{q}+\Delta i_{q}, \omega+\Delta \omega\right)$ which will continue evolving as shown in Figure 11. It is easy to observe that the system will quickly settle to the stable state again, which demonstrates the better disturbance rejection ability.

\section{Conclusions}

In this paper, we have investigated the fixed-time stability of uncertain permanent magnet synchronous motors. We proposed an effective adaptive controller which can stabilize the 


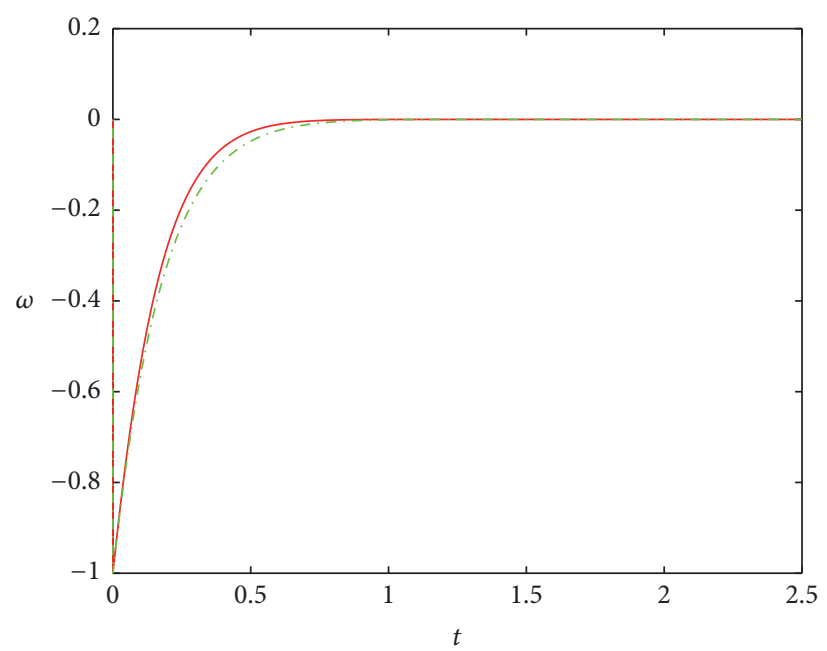

Fixed time

- Finite time

FIGURE 7: Comparison of convergent speed of $\omega$ in fixed time and finite time, respectively.

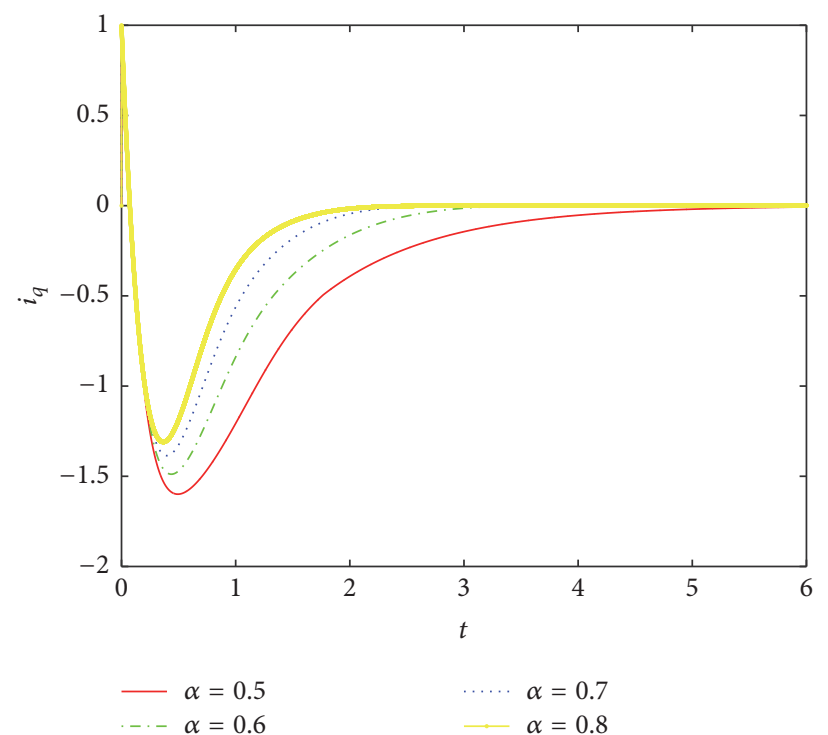

FIGURE 8: The variations of convergent speed of $i_{q}$ with $\beta=1.5, \alpha=0.5,0.6,0.7,0.8$.

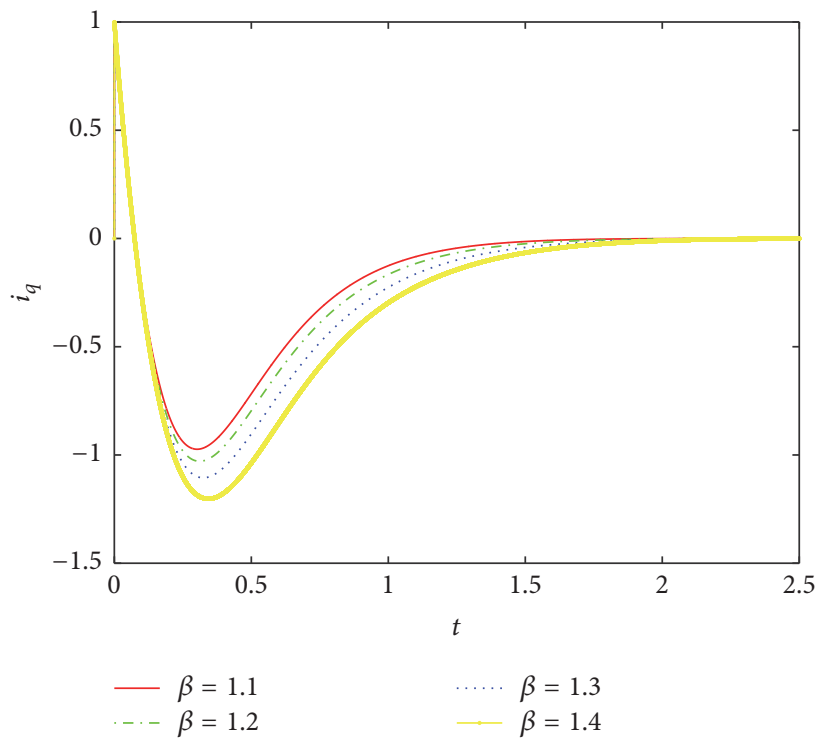

FIGURE 9: The variations of convergent speed of $i_{q}$ with $\alpha=7 / 9, \beta=1.1,1.2,1.3,1.4$. 


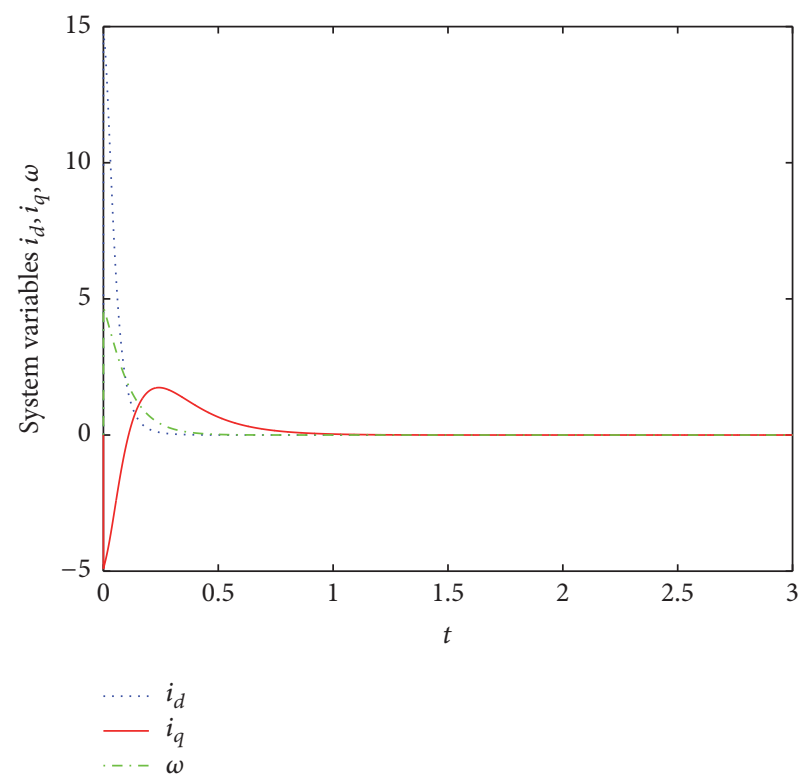

(a)

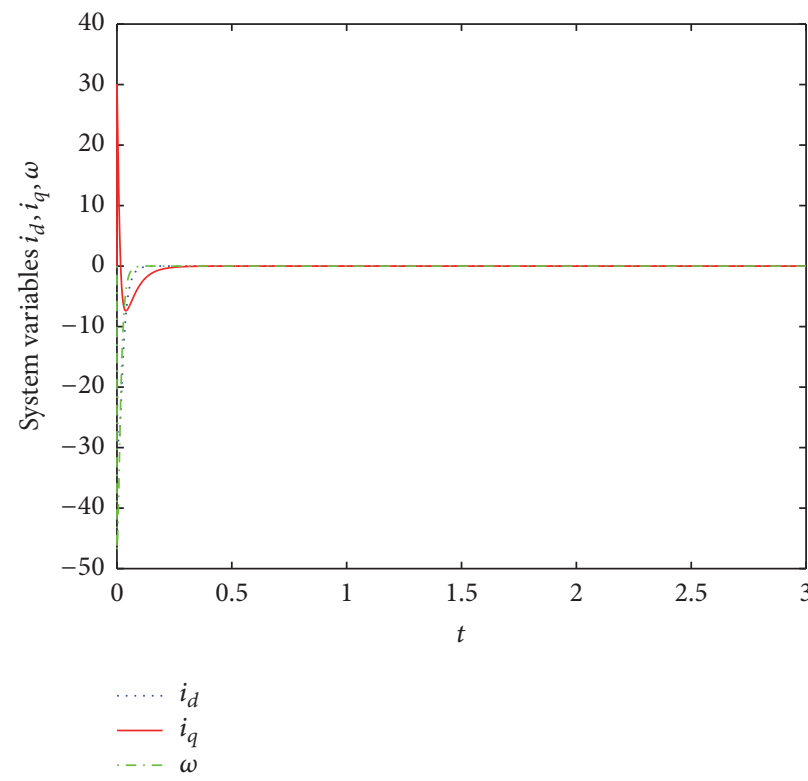

(c)

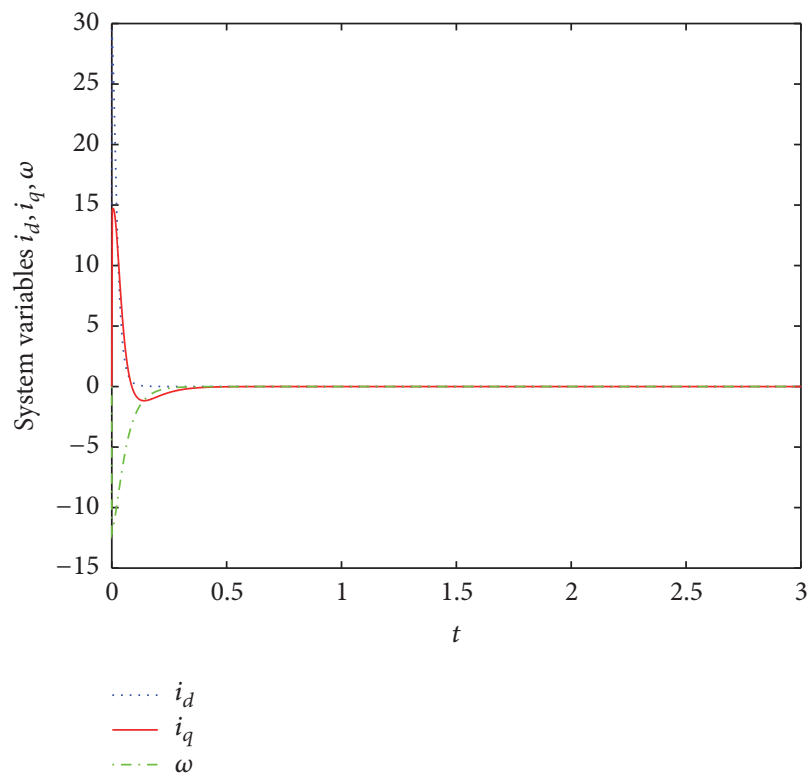

(b)

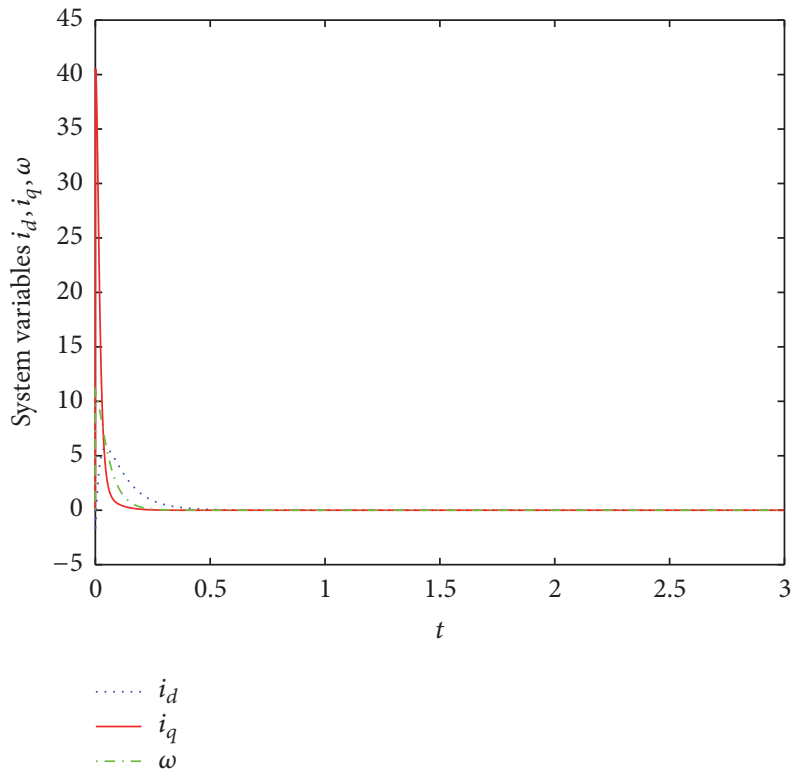

(d)

FIGURE 10: Time response of the controlled state variables $i_{d}, i_{q}$, and $\omega$ by taking the initial states $i_{d}(0), i_{q}(0), \omega(0)$ randomly from the larger interval $[-50,50]$ for four times (a)-(d).

permanent magnet synchronous motors in fixed time based on the Lyapunov stability theory. In comparison with previous methods, the convergent speed of our proposed fixedtime controlling scheme, where the settling time of fixedtime stability could be adjusted to desired values regardless of initial conditions, is faster than that in finite-time controlling scheme. Numerical simulations were provided to illustrate the effectiveness and feasibility of the above results. In the future work, we will further explore the drive-response fixedtime synchronization, parameters identification of uncertain permanent magnet synchronous motors, and the trade-off between controlling time and energy consumption [39]. In addition, because noise perturbation is ubiquitous [40], the present study did not consider the effect of noise perturbation, which is our other research topic.

\section{Conflicts of Interest}

The authors declare that there are no conflicts of interest regarding the publication of this paper.

\section{Acknowledgments}

Maoxing Liu is supported by the National Sciences Foundation of China (11571324, 11301491), the National Sciences Foundation of Shanxi Province (2015011009, 201601D021015), 


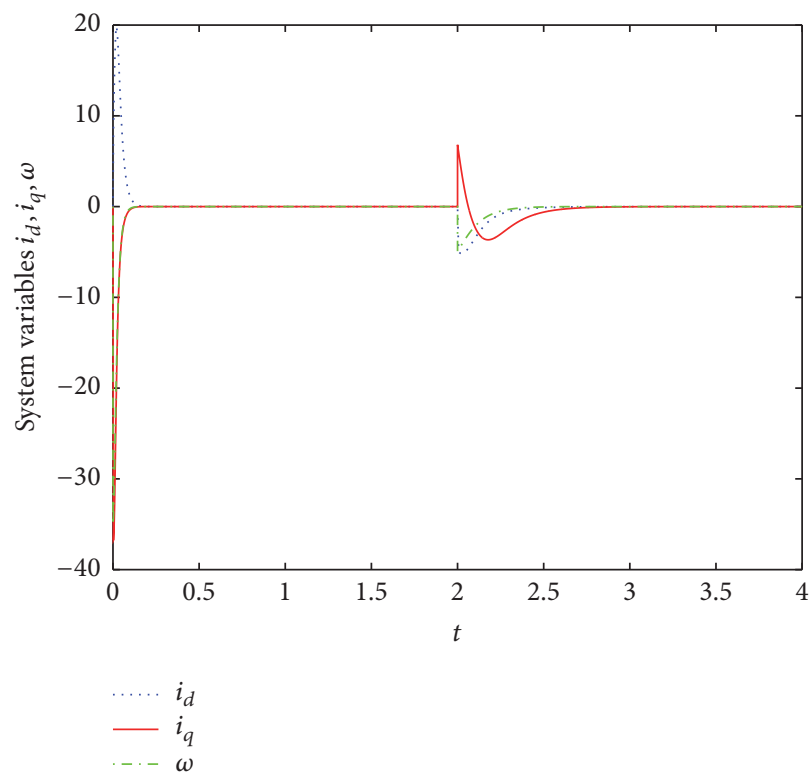

(a)

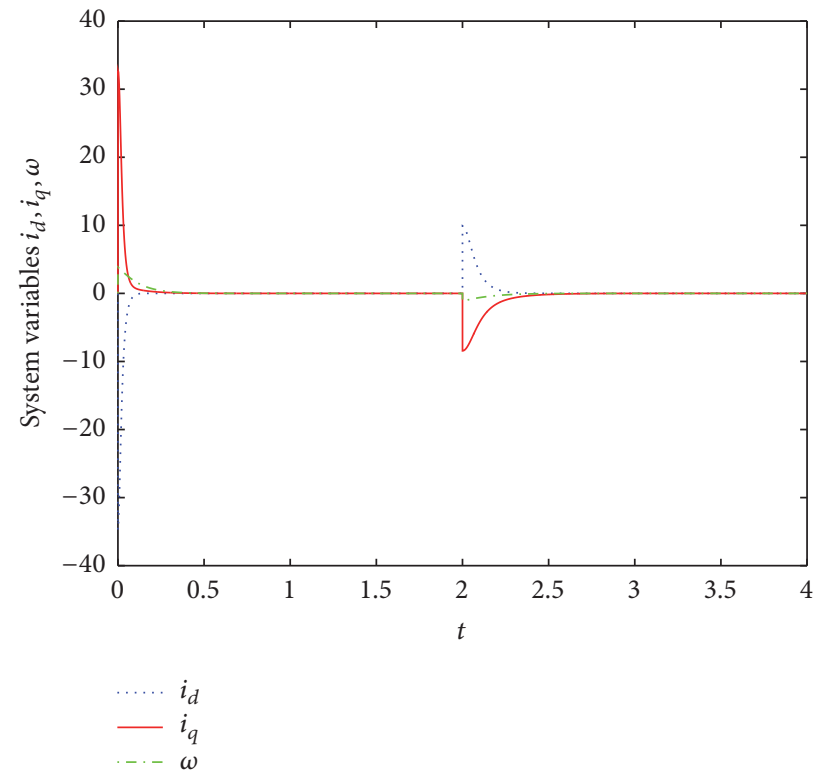

(b)

FIGURE 11: Time response of the controlled state variables $i_{d}, i_{q}$, and $\omega$ suffering from disturbance at time $t=2$.

and the Scientific Activities of Selected Returned Overseas Professionals in Shanxi Province; Yong-zheng Sun is supported by the National Sciences Foundation of China (61403393).

\section{References}

[1] E. N. Lorenz, "Deterministic nonperiodic flow," Journal of the Atmospheric Sciences, vol. 20, no. 2, pp. 130-141, 1963.

[2] T. Shinbrot, E. Ott, C. Grebogi, and J. A. Yorke, "Using chaos to direct trajectories to targets," Physical Review Letters, vol. 65, no. 26, pp. 3215-3218, 1990.

[3] T. Shinbrot, W. Ditto, C. Grebogi, E. Ott, M. Spano, and J. A. Yorke, "Using the sensitive dependence of chaos (the butterfly effect) to direct trajectories in an experimental chaotic system," Physical Review Letters, vol. 68, no. 19, pp. 2863-2866, 1992.

[4] J. D. Skufca, J. A. Yorke, and B. Eckhardt, "Edge of chaos in a parallel shear flow," Physical Review Letters, vol. 96, no. 17, Article ID 174101, 2006.

[5] L. M. Pecora and T. L. Carroll, "Synchronization in chaotic systems," Physical Review Letters, vol. 64, no. 8, pp. 821-824, 1990.

[6] J. Wu, Z.-C. Ma, Y.-Z. Sun, and F. Liu, "Finite-time synchronization of chaotic systems with noise perturbation," Kybernetika, vol. 54, no. 1, pp. 137-149, 2015.

[7] S. Boccaletti, J. Kurths, G. Osipov, D. L. Valladares, and C. S. Zhou, "The synchronization of chaotic systems," Physics Reports, vol. 366, no. 1-2, pp. 1-101, 2002.

[8] J. M. Ottino, F. J. Muzzio, M. Tjahjadi, J. G. Franjione, S. C. Jana, and H. A. Kusch, "Chaos, symmetry, and self-similarity: exploiting order and disorder in mixing processes," Science, vol. 257, no. 5071, pp. 754-760, 1992.

[9] S. J. Schiff, K. Jerger, D. H. Duong, T. Chang, M. L. Spano, and W. L. Ditto, "Controlling chaos in the brain," Nature, vol. 370, no. 6491, pp. 615-620, 1994.
[10] S. Nakata, T. Miyata, N. Ojima, and K. Yoshikawa, "Selfsynchronization in coupled salt-water oscillators," Physica D: Nonlinear Phenomena, vol. 115, no. 3-4, pp. 313-320, 1998.

[11] Y. Kuroe and S. Hayashi, "Analysis of bifurcation in power electronic induction motor drive systems," in Proceedings of the 20th Annual IEEE Power Electronics Specialists Conference, pp. 923-930, Milwaukee, WI, USA.

[12] N. Hemati, "Strange attractors in brushless DC motors," IEEE Transactions on Circuits and Systems I: Fundamental Theory and Applications, vol. 41, no. 1, pp. 40-45, 1994.

[13] Z. Li, J. B. Park, Y. H. Joo, B. Zhang, and G. Chen, "Bifurcations and chaos in a permanent-magnet synchronous motor," IEEE Transactions on Circuits and Systems I: Fundamental Theory and Applications, vol. 49, no. 3, pp. 383-387, 2002.

[14] R. He and Q. Han, "Dynamics and stability of permanentmagnet synchronous motor," Mathematical Problems in Engineering, vol. 2017, Article ID 4923987, 8 pages, 2017.

[15] R. Tao, J. Ma, and H. Zhao, "Torque ripple minimization in PMSM based on an indirect adaptive ROBust controller," Mathematical Problems in Engineering, vol. 2017, Article ID 9512351, 10 pages, 2017.

[16] M. Zribi, A. Oteafy, and N. Smaoui, "Controlling chaos in the permanent magnet synchronous motor," Chaos, Solitons \& Fractals, vol. 41, no. 3, pp. 1266-1276, 2009.

[17] M. Ataei, A. Kiyoumarsi, and B. Ghorbani, "Control of chaos in permanent magnet synchronous motor by using optimal Lyapunov exponents placement," Physics Letters A, vol. 374, no. 41, pp. 4226-4230, 2010.

[18] A. M. Harb, "Nonlinear chaos control in a permanent magnet reluctance machine," Chaos, Solitons \& Fractals, vol. 19, no. 5, pp. 1217-1224, 2004.

[19] H. H. Choi, "Adaptive control of a chaotic permanent magnet synchronous motor," Nonlinear Dynamics, vol. 69, no. 3, pp. 1311-1322, 2012.

[20] G. Maeng and H. H. Choi, "Adaptive sliding mode control of a chaotic nonsmooth-air-gap permanent magnet synchronous 
motor with uncertainties," Nonlinear Dynamics, vol. 74, no. 3, pp. 571-580, 2013.

[21] A. Loria, "Robust linear control of (chaotic) permanent-magnet synchronous motors with uncertainties," IEEE Transactions on Circuits and Systems I: Regular Papers, vol. 56, no. 9, pp. 21092122, 2009.

[22] A. Loria, G. Espinosa-Pérez, and S. Avila-Becerril, "Global adaptive linear control of the permanent-magnet synchronous motor," International Journal of Adaptive Control and Signal Processing, vol. 28, no. 10, pp. 971-986, 2014.

[23] Y. Yu, X. Guo, and Z. Mi, "Adaptive Robust Backstepping Control of Permanent Magnet Synchronous Motor Chaotic System with Fully Unknown Parameters and External Disturbances," Mathematical Problems in Engineering, vol. 2016, Article ID 3690240, 2016.

[24] X. Wu, H. Wang, X. Yuan, S. Huang, and D. Luo, "Design and implementation of recursive model predictive control for permanent magnet synchronous motor drives," Mathematical Problems in Engineering, vol. 2015, Article ID 431734, 2015.

[25] Y. Zhang and X.-F. Cheng, "Sensorless control of permanent magnet synchronous motors and EKF parameter tuning research," Mathematical Problems in Engineering, vol. 2016, Article ID 3916231, 2016.

[26] J. Qian, A. Xiong, and W. Ma, "Extended state observerbased sliding mode control with new reaching law for PMSM speed control," Mathematical Problems in Engineering, vol. 2016, Article ID 6058981, 2016.

[27] W. Du-Qu and Z. Bo, "Controlling chaos in permanent magnet synchronous motor based on finite-time stability theory," Chinese Physics B, vol. 18, no. 4, pp. 1399-1403, 2009.

[28] C.-S. Tang and Y.-H. Dai, "Finite-time stability control of permanent magnet synchronous motor chaotic system with parameters uncertain," Wuli Xuebao/Acta Physica Sinica, vol. 62, no. 18, Article ID 180504, 2013.

[29] J. Wang, X. Chen, and J. Fu, "Adaptive finite-time control of chaos in permanent magnet synchronous motor with uncertain parameters," Nonlinear Dynamics, vol. 78, no. 2, pp. 1321-1328, 2014.

[30] Q. Chen, X. M. Ren, and J. Na, "Robust finite-time chaos synchronization of uncertain permanent magnet synchronous motors," ISA Transactions $^{\circledR}$, vol. 58, no. 1, pp. 262-269, 2015.

[31] Y. Sun, X. Wu, L. Bai, Z. Wei, and G. Sun, "Finite-time synchronization control and parameter identification of uncertain permanent magnet synchronous motor," Neurocomputing, 2015.

[32] H. Du, G. Wen, Y. Cheng, Y. He, and R. Jia, "Distributed FiniteTime Cooperative Control of Multiple High-Order Nonholonomic Mobile Robots," IEEE Transactions on Neural Networks and Learning Systems, vol. 28, no. 12, pp. 2998-3006, 2017.

[33] H. B. Du, Y. G. He, and Y. Y. Cheng, "Finite-time synchronization of a class of second order non-linear multi-agent systems using output feedback control," IEEE Transactions on Circuits and Systems II: Express Briefs, vol. 61, no. 6, pp. 1778-1788, 2014.

[34] A. Polyakov, "Nonlinear feedback design for fixed-time stabilization of linear control systems," Institute of Electrical and Electronics Engineers Transactions on Automatic Control, vol. 57, no. 8, pp. 2106-2110, 2012.

[35] S. E. Parsegov, A. E. Polyakov, and P. S. Shcherbakov, "Fixedtime consensus algorithm for multi-agent systems with integrator dynamics," in Proceedings of the 4th IFAC Workshop on Distributed Estimation and Control in Networked Systems, NecSys 2013, pp. 110-115, September 2013.
[36] A. Polyakov, D. Efimov, and W. Perruquetti, "Finite-time and fixed-time stabilization: Implicit Lyapunov function approach," Automatica, vol. 51, pp. 332-340, 2015.

[37] S. P. Bhat and D. S. Bernstein, "Finite-time stability of continuous autonomous systems," SIAM Journal on Control and Optimization, vol. 38, no. 3, pp. 751-766, 2000.

[38] H. K. Khalil and J. W. Grizzle, Nonlinear systems, Upper Saddle River: Prentice Hall, 2002.

[39] Y. Sun, S. Leng, Y. Lai, C. Grebogi, and W. Lin, "Closed-Loop Control of Complex Networks: A Trade-Off between Time and Energy," Physical Review Letters, vol. 119, no. 19, 2017.

[40] R. Erban, J. Haškovec, and Y. Sun, "A Cucker-Smale model with noise and delay," SIAM Journal on Applied Mathematics, vol. 76, no. 4, pp. 1535-1557, 2016. 


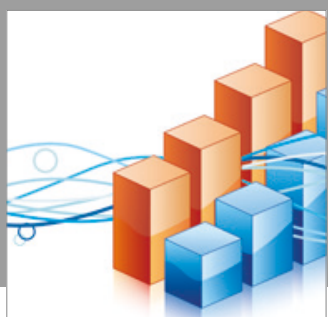

Advances in

Operations Research

vatersals

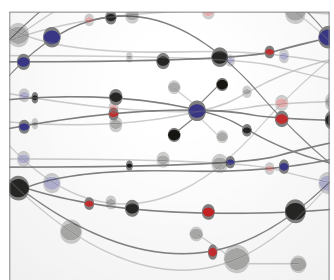

\section{The Scientific} World Journal
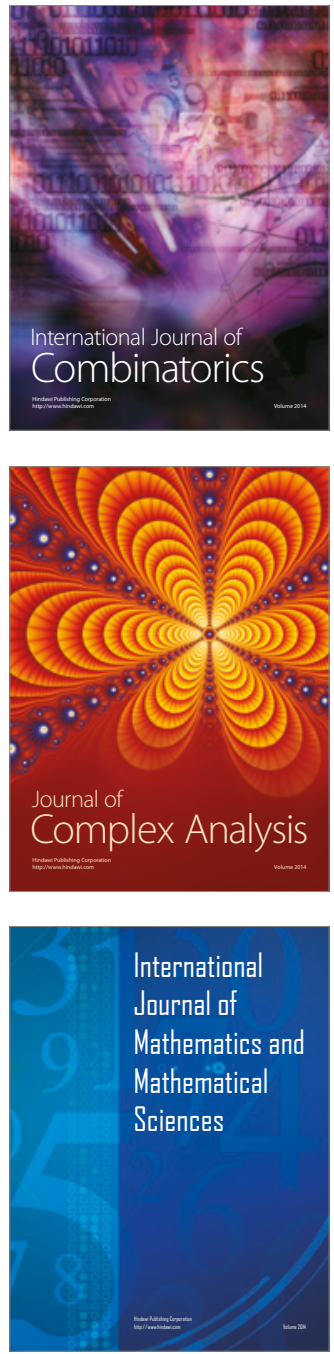
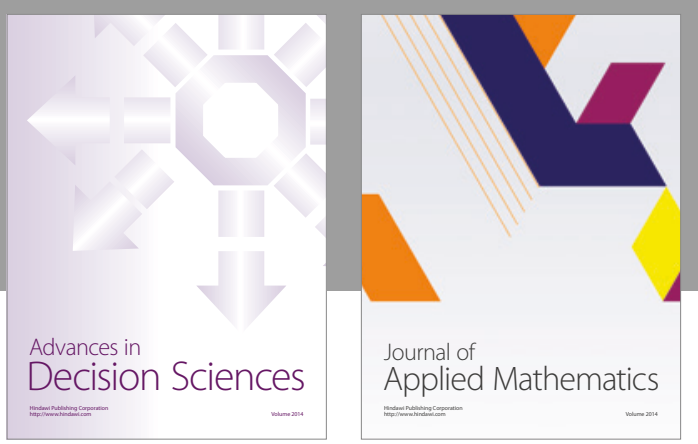

Algebra

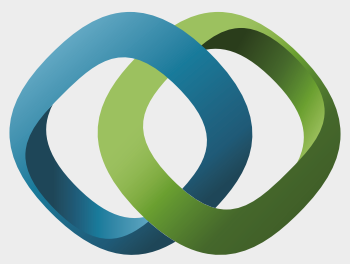

\section{Hindawi}

Submit your manuscripts at

https://www.hindawi.com
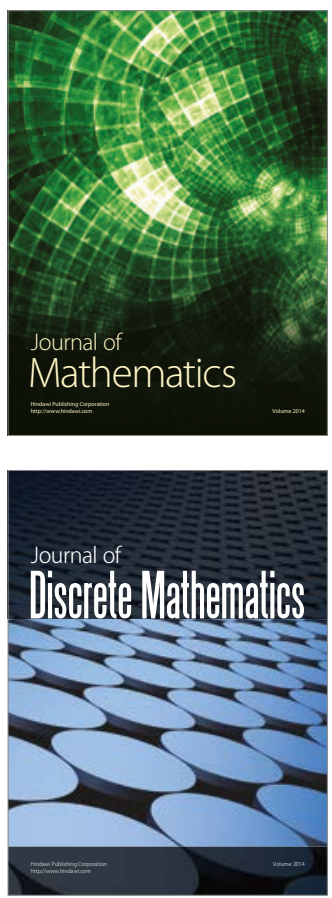

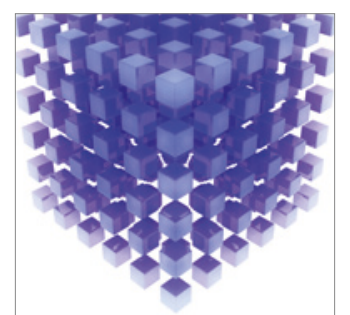

Mathematical Problems in Engineering
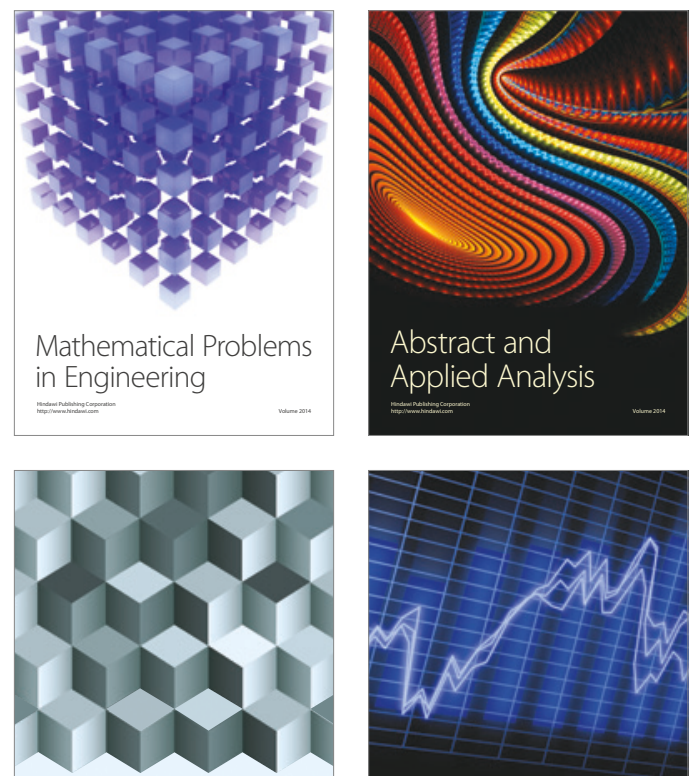

Journal of

Function Spaces

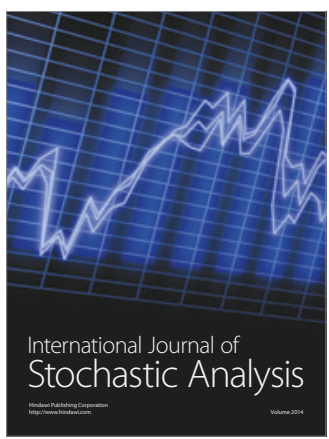

Probability and Statistics
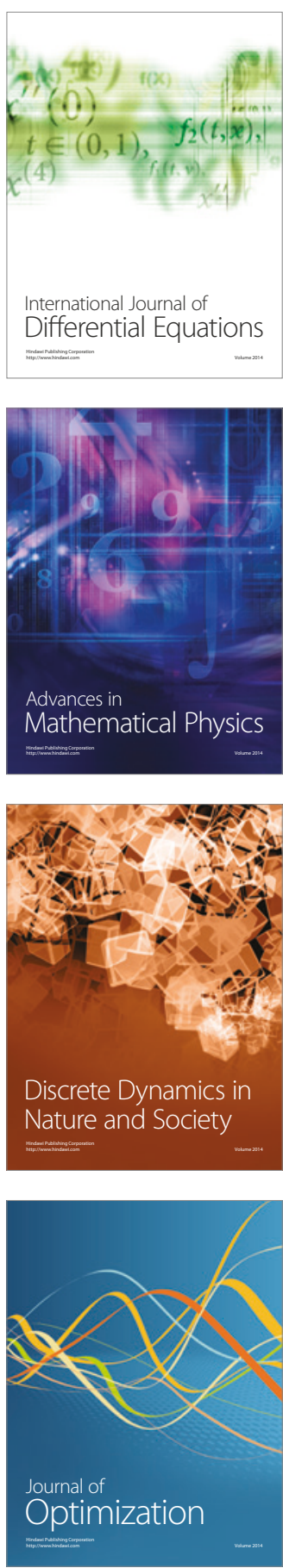